\title{
INIQUIDADES SOCIOECONÔMICAS NA SAÚDE BUCAL DE ESTUDANTES UNIVERSITÁRIOS DO SUL DO BRASIL
}

\author{
Socioeconomic inequities in the oral health \\ of university students in southern Brazil
}

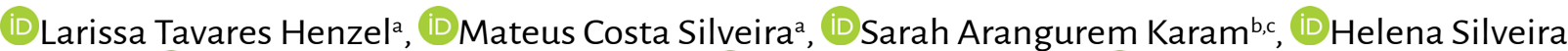
Schuch $^{\mathrm{b}}$ (D) Mariana Gonzalez Cademartorib , (D) Marcos Britto Corrêa ${ }^{\mathrm{b}}$, (D)Flávio Fernando Demarco ${ }^{\mathrm{b}, \mathrm{c}}$
\end{abstract}

\section{RESUMO}

Objetivo: Identificar a magnitude da associação entre experiência de cárie dentária e autopercepção negativa de saúde bucal com determinantes socioeconômicos. Métodos: Estudo transversal realizado com dados de uma coorte prospectiva com os universitários ingressantes na Universidade Federal de Pelotas (UFPel) no ano de 2016. Os dados foram coletados por meio de questionário autoaplicável, incluindo características demográficas, socioeconômicas e psicossociais. Os desfechos do presente estudo foram a experiência de cárie autorrelatada (histórico de doença cárie) e autopercepção de saúde bucal (positiva versus negativa). Resultados: Um total de 3.237 alunos ingressou, dos quais 2.089 (64,5\%) concordaram em participar do estudo. O modelo de regressão de Poisson mostrou que universitários com renda familiar de R\$ 1001,00 a 5000,00 e R\$ 5001,00 ou mais apresentaram, respectivamente, uma razão de prevalência (RP) 14\% $(\mathrm{RP}=0,86$; IC95\% 0,80-0,92) e 18\% (RP = 0,82; IC95\% 0,74 a 0,90$)$ menor de experiência de cárie, assim como indivíduos cujas mães tinham ensino médio completo apresentaram uma prevalência $14 \%$ menor $(R P=0,86$; IC95\% $0,80$ a 0,92$)$ e ensino superior completo $19 \%(R P=0,81$; IC95\% 0,75 a 0,87) menor de experiência de cárie, quando comparados aos grupos de referência. Na autopercepção de saúde bucal, os resultados para renda familiar de $\mathrm{R} \$ 1001$ a 5000,00 e R\$ 5001 ou mais apresentaram, respectivamente, uma prevalência $23 \%(R P=0,77$; IC95\% 0,64 a 0,91) e 43\% (RP = 0,57; IC95\% 0,45 a 0,72) menor de ter autopercepção de saúde bucal negativa e indivíduos cujas mães tinham ensino superior completo reportaram uma prevalência $21 \%$ menor de autopercepção de saúde bucal negativa quando comparados à referência $(\mathrm{RP}=0,79$; IC95\% 0,66 a 0,97). Conclusões: Os achados do presente estudo confirmam que os indicadores socioeconômicos influenciam a experiência de cárie autorrelatada e a autopercepção de saúde bucal dos universitários.

Palavras-chave: Determinantes sociais da saúde. Inequidade social. Cárie dentária. Saúde bucal.

\section{ABSTRACT}

Objective: To identify the magnitude of the association between dental caries experience and negative self-perception of oral health with socioeconomic determinants. Methods: Cross-sectional study conducted with data from a prospective cohort with university students entering the Federal University of Pelotas (UFPel) in 2016. Data were collected through a self-administered questionnaire, including demographic, socioeconomic and psychosocial characteristics. The outcomes of the present study were experience of self-reported caries (history of caries disease) and self-perceived oral health (positive versus negative). Results: A total of 3,237 students joined, of which 2,089 (64.5\%) agreed to participate in the study. The Poisson regression model known that university students with an income of R\$ 1001 to 5000.00 and R\$ 5001 or more primary, respectively, a prevalence ratio $(\mathrm{PR}) 14 \%(\mathrm{PR}=0,86 ; 95 \% \mathrm{CI} 0,80-0,92)$ and $18 \%(\mathrm{PR}=0,82$; $95 \%$ CI 0,74 a 0,90$)$ lower of caries experience, as well as 
individualizing mothers had completed high school prevalence a 14\% (PR =0,86; 95\%CI 0,80 a 0,92$)$ lower prevalence and complete higher education $19 \%(\mathrm{PR}=0,81 ; 95 \% \mathrm{CI} 0,75$ a 0,87$)$ less caries experience when compared to reference groups. In the self-perception of oral health, the results for income of R\$ 1001 to 5000.00 and R\$ 5001 or more dissipated, respectively, a 23\% (PR = 0,77; 95\%CI 0,64 a 0,91) and $43 \%(\mathrm{PR}=0,57$; 95\%CI 0,45 a 0,72$)$ lower prevalence of having negative self-perception of oral health and qualified originating from complete higher education reported a $23 \%$ lower prevalence of negative self-perceived oral health when compared to the reference ( $\mathrm{PR}=0,79 ; 95 \% \mathrm{CI} 0,66$ a 0,97 ). Conclusions: The findings of the present study confirm that socioeconomic indicators influence the experience of caries and self-perceived oral health among university students.

Keywords: Social determinants of health. Social inequity. Dental caries. Oral health.

\section{INTRODUÇÃO}

Os agravos em saúde bucal são um problema de saúde pública mundial que, apesar de serem passíveis de prevenção, ainda possuem uma alta prevalência' ${ }^{1}$ A cárie dentária não tratada em dentes permanentes foi a condição de saúde mais prevalente do mundo no ano de 2017, afetando cerca de 2,3 bilhões de pessoas ${ }^{2}$. As lesões de cárie dependem da ingestão de uma dieta rica em açúcares livres e higiene bucal inadequada, porém é definida como uma doença multifatorial, pois ocorre devido a fatores simultâneos, sendo uma infecção crônica de progressão lenta causada por fatores biológicos, comportamentais e sociais ${ }^{3,4}$.

Pesquisas sobre a influência dos determinantes sociais nas doenças bucais têm alcançado grande importância no campo científico, abrangendo fatores socioeconômicos, como a renda familiar ou individual, a ocupação/profissão dos indivíduos e o grau de escolaridade ${ }^{5,6}$. Estudos relatam associação entre posição socioeconômica e desfechos de saúde bucal, como experiência de cárie e doença periodontal ${ }^{7,8}$. Segundo a Comissão Nacional sobre Determinantes Sociais da Saúde ${ }^{9}$, as condições culturais, ambientais e socioeconômicas de uma população geram uma estratificação em diferentes posições sociais dos indivíduos, apresentando relação direta com as condições de saúde ${ }^{9}$. Condições como a base educacional do indivíduo ou dos pais pode interferir no acesso a informação e nos comportamentos em saúde, interferindo na higiene bucal, nos hábitos alimentares e no padrão de utilização de serviços de saúde ${ }^{10-12}$.

As desigualdades socioeconômicas estão associadas ao estado de saúde bucal, seja objetivamente (doenças bucais clinicamente diagnosticadas) ou subjetivamente por meio da autopercepção de saúde bucal ${ }^{5,8,13-16}$. Geralmente estima-se essa associação, condições de saúde bucal e fatores socioeconômicos, através da utilização de um tipo de indicador de doença bucal, preferencialmente os objetivos, sendo assim menos preterido pelos estudos abordar desfechos subjetivos em conjunto aos objetivos ${ }^{17}$. Porém, é notável nas avaliações subjetivas a existência das influências culturais, sociais e psicossociais na saúde bucal ${ }^{18}$.

O complexo âmbito das desigualdades socioeconômicas dificultam a prioridade do cuidado à saúde bucal e o acesso aos serviços odontológicos, gerando consequências à saúde bucal, que demonstram a polarização da doença cárie nos estratos mais pobres da população $0^{1,6,10,19}$. Apesar de diversas pesquisas demonstrarem que os determinantes sociais existentes contribuem para a etiologia da cárie, são necessárias pesquisas com populações distintas para demonstrar a indispensabilidade de políticas públicas voltadas para a redução das iniquidades ${ }^{7}$. Neste sentido, este estudo busca avaliar a magnitude da associação entre experiência de cárie dentária e autopercepção negativa de saúde bucal em relação aos determinantes socioeconômicos (renda familiar, escolaridade materna e atividade remunerada). 


\section{MATERIAIS E MÉTODOS}

Estudo transversal descritivo realizado com dados de uma coorte prospectiva com os universitários ingressantes na Universidade Federal de Pelotas (UFPel) no ano de 2016. Pelotas é uma cidade localizada no sul do Rio Grande do Sul, com uma população de aproximadamente 350.000 pessoas, considerada um dos principais pólos educacionais do sul do Brasil. A UFPel conta com aproximadamente 18.000 alunos matriculados, sendo a única universidade pública da cidade.

Os acadêmicos que ingressaram no primeiro semestre em 2016 na UFPel foram elegíveis ao estudo. Foram excluídos os estudantes que se encontravam em sala de aula durante a aplicação do questionário, mas encontravam-se em regime de matrícula não-regular, eram pertencentes a outros semestres letivos e/ou eram alunos incapazes de realizar o autopreenchimento do questionário.

Foi realizado o cálculo amostral considerando a estimativa do número de ingressantes no primeiro semestre de 2016 (3.000 alunos), utilizando parâmetros para estimativa de prevalência (50\%) e associação (poder de $80 \%$ e $\alpha=5 \%$ ), sendo o tamanho mínimo da amostra necessário de 614 indivíduos. Visando uma variabilidade proporcional dos cursos, deveriam ser entrevistados pelo menos $60 \%$ dos alunos de cada curso de graduação presencial da UFPel, assim, os cursos foram visitados até atingir o tamanho mínimo da amostra de $60 \%$ do total de alunos matriculados.

A equipe de campo foi formada por 25 pessoas, entre alunos de graduação e pós-graduação da Faculdade de Odontologia da UFPEL, a qual participou de um treinamento teórico-prático prévio com duração de quatro horas. O projeto foi aprovado pelo Comitê de Ética em Pesquisa em Seres Humanos da Faculdade de Medicina da Universidade Federal de Pelotas (protocolo número 49449415.2.0000.5317). Todos os participantes foram previamente informados sobre os objetivos do estudo e a confidencialidade de suas respostas, sendo em seguida convidados a assinarem um termo de consentimento livre e esclarecido.

Foram utilizados dois questionários autoaplicados, previamente testados em um estudo piloto com 100 universitários $(n=100)$ matriculados no segundo semestre do curso de graduação, não elegíveis para o estudo, e pertencentes a cinco unidades acadêmicas diferentes da UFPEL que foram selecionadas aleatoriamente. O primeiro questionário continha questões sobre condições sociodemográficas, comportamentais, psicossociais, e de saúde bucal dos indivíduos, enquanto o segundo questionário era anônimo e se referia ao uso de tabaco, drogas e outras substâncias. Os questionários foram aplicados ao longo dos primeiros seis meses do ano de 2016, em sala de aula, durante o horário curricular com autorização prévia do professor.

O desfecho objetivo do presente estudo, a experiência de cárie, foi mensurado através da pergunta "Você tem ou já teve algum dente afetado pela cárie dentária?"20 com opções de resposta sim e não. A autopercepção de saúde bucal, desfecho subjetivo, foi mensurada pela pergunta "Comparando com as pessoas da sua idade, você considera a saúde dos seus dentes, da boca e das gengivas", com as respostas dicotomizadas em autopercepção positiva (muito boa e boa) e negativa (regular, muito ruim e ruim).

As co-variáveis avaliadas referentes às características socioeconômicas foram: renda familiar, escolaridade materna e atividade remunerada. Renda familiar foi medida em categorias pré-definidas em reais: até 500,00; 501,00 até 1.000,00; 1,001,00 até 2.500,00; 2.501,00 até 5.000,00; 5.001,00 até 10.000,00; mais de 10.001 reais. Escolaridade materna foi acessada em categorias, avaliando o maior grau de ensino obtido: sem estudo, ensino fundamental incompleto, ensino fundamental completo, ensino médio incompleto, ensino médio completo, ensino superior incompleto, ensino superior completo. Por fim, a situação empregatícia foi 
avaliada através da pergunta "Você exerce alguma atividade remunerada?", com opções de resposta "sim" e "não".

As variáveis incluídas como ajuste foram sexo (masculino/feminino), idade (em anos completos) e cor da pele. A cor da pele foi coletada seguindo a abordagem do Instituto Brasileiro de Geografia e Estatística (IBGE): branca, preta, parda, amarela, indígena e dicotomizada em grupo majoritário (autodeclarados cor da pele branca) e grupo minoritário (autodeclarados cor da pele preta/parda/amarela/indígena).

Maiores informações sobre a metodologia da pesquisa podem ser consultadas através do artigo metodológico ${ }^{21}$.

\section{ANÁlise ESTATÍSTICA}

Os dados foram inseridos em planilha Office do Excel (Microsoft Office) por digitação dupla, visando detectar possíveis falhas, sendo realizadas verificações automáticas para a faixa de consistência e validação de arquivos duplicados. A análise dos dados foi realizada no Stata 15.0 (Stata Corp., College Station, EUA). Para os dados faltantes, foi realizada a técnica de imputação múltipla por modelo de equações em cadeia assumindo uma distribuição aleatória para os dados faltantes ${ }^{22}$. Foi realizada uma análise descritiva para estimar as frequências absolutas e relativas das variáveis de interesse deste estudo utilizando o teste de qui-quadrado de Pearson. Nas análises bruta e ajustada, para mensuração da razão de prevalência (RP), utilizou-se a Regressão de Poisson com variância robusta. As variáveis de ajuste foram sexo, cor da pele e idade, possíveis confundidores da associação entre fatores socioeconômicos e condição de saúde bucal ${ }^{1}$. As medidas de efeito foram estimadas com intervalos de confiança de $95 \%$.

\section{RESULTADOS}

Um total de 3.237 alunos ingressaram na Universidade Federal no primeiro semestre de 2016, dos quais 2.089 (64,5\%) concordaram em participar do estudo. Destes participantes, mais da metade eram do sexo feminino (52,2\%), com idade entre 18 a 24 anos $(66,1 \%)$ e com renda familiar entre $\mathrm{R} \$ 1.001,00$ e $5.000,00$ (60,8\%). 74,0\% reportaram cor de pele branca, $77,3 \%$ relataram não ter nenhuma atividade remunerada e 32,3\% tinham mães com ensino superior completo. 68,2\% relataram ter tido experiência de cárie durante a vida e 71,4\% apresentaram autopercepção de saúde bucal positiva. A Tabela 1 apresenta as características da amostra. 
Tabela 1: Descrição das características da amostra. Pelotas/Brasil 2016.

\begin{tabular}{|c|c|c|}
\hline Variáveis & $\%$ & IC 95\% \\
\hline \multicolumn{3}{|l|}{ Sexo } \\
\hline Masculino & 47,8 & $45,6-49,9$ \\
\hline Feminino & 52,2 & $50,1-54,4$ \\
\hline \multicolumn{3}{|l|}{ Grupos cor da pele } \\
\hline Majoritário (branca) & 74,0 & $72,1-75,9$ \\
\hline Minoritário (preta/parda/amarela/indígena) & 26,0 & $24,1-27,9$ \\
\hline \multicolumn{3}{|l|}{ Idade } \\
\hline 16-17 anos & 15,0 & $13,4-16,5$ \\
\hline 18-24 anos & 66,1 & $64,1-68,1$ \\
\hline 25-34 anos & 10,3 & $9,0-11,6$ \\
\hline 35 ou mais & 8,6 & $7,4-9,8$ \\
\hline \multicolumn{3}{|l|}{ Renda familiar } \\
\hline$\leq 1000,00$ & 16,0 & $14,2-17,7$ \\
\hline 1001,00 a 5000,00 & 60,8 & $58,5-63,2$ \\
\hline$\geq 5001,00$ & 23,2 & $21,0-25,4$ \\
\hline \multicolumn{3}{|l|}{ Atividade remunerada } \\
\hline Não & 77,3 & $75,5-79,1$ \\
\hline Sim & 22,7 & $20,9-24,5$ \\
\hline \multicolumn{3}{|l|}{ Escolaridade materna } \\
\hline Não estudou ou ens. fundamental incompleto & 22,8 & $21,0-24,6$ \\
\hline Ens. fundamental completo & 13,3 & $11,8-14,7$ \\
\hline Ens. médio completo & 31,6 & $29,5-33,6$ \\
\hline Ens. superior completo & 32,3 & $30,3-34,4$ \\
\hline \multicolumn{3}{|l|}{ Experiência de cárie dentária autorrelatada } \\
\hline Não & 31,8 & $29,8-33,8$ \\
\hline Sim & 68,2 & $66,2-70,2$ \\
\hline \multicolumn{3}{|l|}{ Autopercepção de saúde bucal } \\
\hline Positiva & 71,4 & $69,4-73,3$ \\
\hline Negativa & 28,6 & $26,7-30,6$ \\
\hline
\end{tabular}


Na tabela 2, observa-se que a experiência de cárie autorrelatada foi associada a indivíduos com idade mais elevada, do sexo feminino, com menor renda familiar, filhos de mães com menor escolaridade, que exerciam atividade remunerada e não foi associada à cor da pele. Em relação à autopercepção de saúde bucal (Tabela 3), foi observada associação com o grupo minoritário de cor da pele, sexo masculino, idade mais elevada, menor renda familiar, indivíduos com mães com menor escolaridade e a indivíduos que exerciam atividade remunerada.

Tabela 2: Análise bivariada entre variáveis socioeconômicas e experiência de cárie autorrelatada com chi-quadrado de Pearson. Pelotas/Brasil, 2016.

\section{Experiência de cárie}

\section{Não \% (IC95\%) Sim \%(IC95\%)}

\section{Sexo}

Masculino

$36,3(33,3-39,3)$

$63,7(60,6-66,7)$

Feminino

$27,6(24,9-30,3)$

$72,4(69,7-75,1)$

\section{Grupos cor da pele}

Majoritário (branca)

$31,7(29,3-33,9)$

$68,3(66,0-70,1)$

Minoritário (preta/parda/amarela/indígena)

$32,1(28,1-36,1)$

$67,9(63,9-71,9)$

\section{Idade}

16-17 anos

$43,5(38,0-49,1)$

$56,5(50,9-61,9)$

18-24 anos

$35,0(32,5-37,5)$

$65,0(62,5-67,5)$

25-34 anos

$18,5(13,3-23,7)$

$81,5(76,3-86,6)$

35 ou mais

$2,3(0,6-4,5)$

$97,7(95,5-99,9)$

\section{Renda familiar}

$\leq 1000,00$

1001,00 a 5000,00

$\geq 5001,00$

\section{Atividade remunerada}

Não

$\operatorname{sim}$

\section{Escolaridade materna}

Não estudou ou ens. fundamental incompleto

Ens. fundamental completo

$16,9(13,5-20,3)$

$83,1(79,6-86,5)$

$29,3(23,8-34,7)$

$70,7(65,3-76,2)$

Ens. médio completo

$35,0(31,3-38,6)$

$65,0(61,4-68,7)$

Ens. superior completo

$\begin{array}{ll}20,0(15,1-24,9) & 80,0(75,1-84,8) \\ 32,3(29,5-34,9) & 67,7(65,0-70,4) \\ 38,5(33,8-43,1) & 61,5(56,9-66,1)\end{array}$

$34,5(32,1-36,8)$

$65,5(63,2-67,8)$

$77,4(73,6-81,2)$
$59,8(56,1-63,5)$ 
Tabela 3: Análise bivariada entre variáveis socioeconômicas e autopercepção de saúde bucal com chi-quadrado de Pearson. Pelotas/Brasil, 2016.

\begin{tabular}{|c|c|c|}
\hline & \multicolumn{2}{|c|}{ Autopercepção de saúde bucal } \\
\hline & Positiva \%(IC95\%) & Negativa \%(IC95\%) \\
\hline \multicolumn{3}{|l|}{ Sexo } \\
\hline Masculino & $66,7(63,7-69,6)$ & $33,3(30,4-36,3)$ \\
\hline Feminino & $75,7(73,1-78,2)$ & $24,3(21,8-26,9)$ \\
\hline \multicolumn{3}{|l|}{ Grupos cor da pele } \\
\hline Majoritário (branca) & $74,1(71,8-76,3)$ & $25,9(23,7-28,1)$ \\
\hline Minoritário (preta/parda/ amarela/indígena) & $63,6(59,5-67,7)$ & $36,4(32,3-40,5)$ \\
\hline \multicolumn{3}{|l|}{ Idade } \\
\hline 16-17 anos & $76,6(71,9-81,4)$ & $23,4(18,6-28,1)$ \\
\hline 18-24 anos & $71,9(69,5-74,3)$ & $28,1(25,7-30,5)$ \\
\hline 25-34 anos & $70,7(64,6-76,8)$ & $29,3(23,2-35,4)$ \\
\hline 35 ou mais & $58,8(51,5-66,1)$ & $41,2(33,9-48,5)$ \\
\hline \multicolumn{3}{|l|}{ Renda familiar } \\
\hline$\leq 1000,00$ & $61,1(55,5-66,8)$ & $38,9(33,2-44,5)$ \\
\hline 1001,00 a 5000,00 & $71,2(68,6-73,7)$ & $28,8(26,3-31,4)$ \\
\hline$\geq 5001,00$ & $78,9(74,9-82,9)$ & $21,1(17,1-25,0)$ \\
\hline \multicolumn{3}{|l|}{ Atividade remunerada } \\
\hline Não & $72,7(70,5-74,8)$ & $27,3(25,1-29,5)$ \\
\hline Sim & $67,0(62,7-71,2)$ & $33,0(28,8-37,3)$ \\
\hline \multicolumn{3}{|l|}{ Escolaridade materna } \\
\hline Não estudou ou ens. fundamental incompleto & $66,4(62,1-70,7)$ & $33,6(29,3-37,8)$ \\
\hline Ens. fundamental completo & $68,9(63,4-74,4)$ & $31,1(25,6-36,6)$ \\
\hline Ens. médio completo & $72,0(68,5-75,4)$ & $28,0(24,5-31,5)$ \\
\hline Ens. superior completo & $75,3(71,9-78,6)$ & $24,7(21,4-28,0)$ \\
\hline
\end{tabular}

Na tabela 4, estimativas obtidas por modelos de regressão de Poisson mostraram que universitários com renda familiar de R\$1001,00 a 5000,00 e R\$ 5001,00 ou mais apresentaram, respectivamente, uma razão de prevalência 14\% ( $R P=0,86$; IC95\% 0,80-0,92) e 18\% ( $R P=0,82$; IC95\% 0,74 a 0,90) menor de experiência de cárie autorrelatada, assim como indivíduos cujas mães tinham ensino médio completo apresentaram uma prevalência $14 \%$ menor $(\mathrm{RP}=0,86$; IC95\% 0,80 a 0,92) e ensino superior completo 19\% menor ( $R P=0,81$; IC95\% 0,75 a 0,87) de experiência de cárie autorrelatada, quando comparados aos grupos de referência. Para a autopercepção de saúde bucal, os resultados demostraram que universitários com renda 
familiar de $\mathrm{R} \$ 1001,00$ a 5000,00 e R\$ 5001,00 ou mais apresentaram, respectivamente, uma prevalência 23\% ( $\mathrm{RP}=0,77$; IC95\% 0,64 a 0,91) e 43\% menor $(\mathrm{RP}=0,57$; IC95\% 0,45 a 0,72) de ter autopercepção de saúde bucal negativa e indivíduos cujas mães tinham ensino superior completo reportaram uma prevalência $21 \%$ menor de autopercepção de saúde bucal negativa $(\mathrm{RP}=$ 0,79; IC95\% 0,66 a 0,97) em comparação a indivíduos com renda familiar menor que $\mathrm{R} \$ 1000$ mensais e escolaridade materna até ensino fundamental incompleto, respectivamente.

Tabela 4: Análise de Regressão de Poisson, com razão de prevalência (PR) bruta e ajustada de experiência de cárie autorrelatada e autopercepção de saúde bucal para as variáveis socioeconômicas. Pelotas/Brasil, 2016.

\begin{tabular}{|c|c|c|c|c|}
\hline & \multicolumn{2}{|c|}{ Experiência de cárie } & \multicolumn{2}{|c|}{$\begin{array}{l}\text { Autopercepção negativa } \\
\text { de saúde bucal }\end{array}$} \\
\hline & $\begin{array}{l}\mathbf{R P}_{\text {bruta }} \\
\text { (IC95\%) }\end{array}$ & $\begin{array}{l}\mathbf{R P}_{\text {ajustada" }} \\
\text { (IC95\%) }\end{array}$ & $\begin{array}{l}\mathbf{R P}_{\text {bruta }} \\
\text { (IC95\%) }\end{array}$ & $\begin{array}{l}\text { RP }_{\text {ajustada" }} \\
\text { (IC95\%) }\end{array}$ \\
\hline \multicolumn{5}{|l|}{ Renda familiar } \\
\hline$\leq 1000,00$ & Ref. & Ref. & Ref. & Ref. \\
\hline 1001,00 a 5000,00 & $\begin{array}{l}0,85 \\
(0,78-0,91)\end{array}$ & $\begin{array}{l}0,86 \\
(0,80-0,92)\end{array}$ & $\begin{array}{l}0,74 \\
(0,62-0,88)\end{array}$ & $\begin{array}{l}0,77 \\
(0,64-0,91)\end{array}$ \\
\hline$\geq 5001,00$ & $\begin{array}{l}0,77 \\
(0,70-0,85)\end{array}$ & $\begin{array}{l}0,82 \\
(0,74-0,90)\end{array}$ & $\begin{array}{l}0,54 \\
(0,43-0,69)\end{array}$ & $\begin{array}{l}0,57 \\
(0,45-0,72)\end{array}$ \\
\hline \multicolumn{5}{|l|}{ Atividade remunerada } \\
\hline Não & Ref. & Ref. & Ref. & Ref. \\
\hline $\operatorname{sim}$ & $\begin{array}{l}1,18 \\
(1,11-1,26)\end{array}$ & $\begin{array}{l}1,03 \\
(0,97-1,11)\end{array}$ & $\begin{array}{l}1,21 \\
(1,04-1,40)\end{array}$ & $\begin{array}{l}1,04 \\
(0,88-1,24)\end{array}$ \\
\hline \multicolumn{5}{|l|}{ Escolaridade materna } \\
\hline $\begin{array}{l}\text { Não estudou ou ens. } \\
\text { fundamental incompleto }\end{array}$ & Ref. & Ref. & Ref. & Ref. \\
\hline Ens. fundamental completo & $\begin{array}{l}0,85 \\
(0,78-0,93)\end{array}$ & $\begin{array}{l}0,90 \\
(0,83-0,98)\end{array}$ & $\begin{array}{l}0,93 \\
(0,74-1,15)\end{array}$ & $\begin{array}{l}0,94 \\
(0,75-1,17)\end{array}$ \\
\hline Ens. médio completo & $\begin{array}{l}0,78 \\
(0,73-0,84)\end{array}$ & $\begin{array}{l}0,86 \\
(0,80-0,92)\end{array}$ & $\begin{array}{l}0,83 \\
(0,69-0,99)\end{array}$ & $\begin{array}{l}0,91 \\
(0,75-1,08)\end{array}$ \\
\hline Ens. superior completo & $\begin{array}{l}0,72 \\
(0,67-0,78)\end{array}$ & $\begin{array}{l}0,81 \\
(0,75-0,87)\end{array}$ & $\begin{array}{l}0,74 \\
(0,61-0,88)\end{array}$ & $\begin{array}{l}0,79 \\
(0,66-0,97)\end{array}$ \\
\hline
\end{tabular}

*Ajustado para sexo, cor da pele e idade.

\section{Discussão}

Observou-se associação entre determinantes socioeconômicos em saúde, como renda familiar e escolaridade materna, e desfechos de saúde bucal entre universitários. Uma maior renda familiar e uma maior escolaridade materna mostraram-se como fatores protetivos tanto para experiência de cárie autorrelatada como para a autopercepção negativa de saúde bucal. A relação entre a cárie e a desigualdade social tem sido descrita em diversas pesquisas, sendo 
a desigualdade reconhecida como decisiva no processo saúde-doença, gerando iniquidades em saúde tanto a nível contextual quanto individual ${ }^{12,13,23}$. Não foi observada associação entre situação empregatícia e saúde bucal, após ajustes para características sociodemográficas.

Estudos prévios argumentaram a relação de ocorrência da cárie a uma complexa rede causal, em que o contexto onde o indivíduo está inserido leva a diferentes condições de saúde e mostra que desigualdades socioeconômicas se mantêm como uma importante causa para as doenças bucais, sendo essas iniquidades um dos principais desafios a serem enfrentados em questões de saúde pública ${ }^{23-25}$. Uma revisão sistemática sobre indicadores socioeconômicos e experiência de cárie analisou 155 estudos envolvendo um total de 329,798 participantes e identificou que a experiência de cárie foi significativamente maior naqueles com menor nível educacional e renda própria ou parental mais baixa ${ }^{8}$. Corroborando outros relatos da literatura, os achados dessa pesquisa confirmaram que quanto menor a renda do indivíduo e menor a escolaridade materna, maior a prevalência de experiência de cárie e de autopercepção de saúde bucal negativa ${ }^{10,24,26,27}$.

Pesquisas avaliando a autopercepção do indivíduo são fundamentais na área da saúde, pois a resposta de cada indivíduo é condicionada por suas experiências, meio onde vive, valores culturais e sociais ${ }^{28,29}$. Sendo a autopercepção da saúde bucal influenciada por fatores multidimensionais, como razões sociais, emocionais e econômicas, ela só pode ser compreendida quando a subjetividade dos indivíduos é levada em consideração ${ }^{15}$.

Há evidências que a baixa renda está relacionada com maior prevalência de impactos negativos na saúde bucal, diminuindo o valor atribuído à saúde, impactando na qualidade de vida e acesso à informação sobre cuidados de saúde ${ }^{30,31}$. Além disso, influencia diretamente no modo de viver de cada indivíduo. As condições de vida e de trabalho estratificam, de forma diferenciada, a maneira pela qual as pessoas sentem, pensam e agem a respeito de sua saúde, indo de acordo com nossos resultados, onde quanto menor a renda familiar maior a autopercepção de saúde bucal negativa ${ }^{16,30}$. Entretanto, diferentemente da renda familiar, a realização de uma atividade profissional remunerada conjunta aos estudos acadêmicos não apresentou uma significância com os desfechos de saúde bucal objetivo e subjetivo após ajuste por sexo, cor da pele e idade. Os acadêmicos que possuem um trabalho simultâneo aos estudos conciliam os seus horários em busca de qualificação profissional e aumento da renda mensal ${ }^{32}$. A atividade remunerada pode exercer um impacto positivo na saúde bucal, refletindo os maiores recursos financeiros e qualificação educacional dos trabalhadores nas escolhas de hábitos saudáveis em saúde bucal ${ }^{33}$.

A escolaridade materna está fortemente associada a uma maior experiência de cárie e autopercepção de saúde bucal negativa ${ }^{8,12,34,35}$. Esta associação também foi observada na presente pesquisa, apesar do ajuste para fatores sociodemográficos, o que realça o importante papel da escolaridade da mãe na saúde de seus filhos. O nível de educação é um importante marcador de realidade socioeconômica. Ademais, maiores níveis de escolaridade geralmente são indicadores de maior acesso a informações, melhores empregos, melhores condições de moradia e melhor alimentação $0^{35}$. Um maior nível de escolaridade está associado com uma maior oportunidade de acesso ao conhecimento em saúde ${ }^{11,12,27,36}$. Acredita-se assim que, quando na infância ocorre a convivência com pais de uma escolaridade mais elevada, os indivíduos ficam sujeitos a hábitos de higiene mais saudáveis e a práticas preventivas, influenciando na experiência de cárie por todo o curso da vida ${ }^{12,37}$.

Como limitações, este estudo apresenta o fato de que os dados foram coletados em um mesmo momento, sendo este um estudo com delineamento transversal, não sendo possível inferir relações de causalidade entre as variáveis. Também, a presença de cárie dentária foi autorreportada, sem a realização de um exame clínico, o que pode levar a estimativas não tão precisas de doença. Porém, apresentamos como pontos positivos o fato de que avaliamos diversos indicadores socioeconômicos e demográficos e diferentes indicadores de saúde bucal, 
sendo um objetivo (experiência de cárie autorrelatada) e outro subjetivo (autopercepção) em uma amostra relativamente grande.

Nesse sentido os resultados demonstram que fatores de ordem social devem seguir sendo investigados, principalmente em populações de risco, por influenciarem as condições de saúde bucal. A realização de estudos que integram determinantes contextuais e individuais para explicar a distribuição da cárie dentária vem sendo sugerida como uma estratégia vantajosa para orientar estratégias para a redução das desigualdades em saúde.

\section{CONCLUSÃo}

Os achados do presente estudo confirmam que os indicadores socioeconômicos influenciam a experiência de cárie autorrelatada e a autopercepção de saúde bucal dos universitários. Além disso, os achados mostraram que quanto maior a renda familiar ou escolaridade materna, menor é a possibilidade de experiência de cárie e a autopercepção negativa de saúde bucal dos indivíduos, representando um fator protetivo destes determinantes sociais na saúde bucal. Nesta amostra de universitários, a situação empregatícia parece não influenciar os indicadores de saúde bucal.

\section{CONFLITO DE INTERESSES}

Os autores declaram não haver conflito de interesses.

\section{REFERÊNCIAS}

1. Peres M, Macpherson L, Weyant R, Daly B, Venturelli R, Mathur M, et al. Oral diseases: a global public health challenge. The Lancet. 2019;394(10194): 249-60.

2. Bernabe E, Marcenes W, Hernandez CR, Bailey ], Abreu LG, Alipour V, et al. Global, regional, and national levels and trends in burden of oral conditions from 1990 to 2017: a systematic analysis for the global burden of disease 2017 study. J Dent Res. 2020;99(4):362-73.

3. Selwitz RH, Ismail AI, Pitts NB. Dental caries. The Lancet. 2007; 369(9555):51-9.

4. Sheiham A, James WPT. Diet and dental caries: the pivotal role of free sugars reemphasized. J Dent Res. 2015;94(10):1341-7.

5. Boing AF, Bastos ]L, Peres KG, Antunes ]LF, Peres MA. Social determinants of health and dental caries in Brazil: a systematic review of the literature between 1999 and 2010. Rev Bras de Epidemiol. 2014;17(2):102-15.

6. Boing AF, Peres MA, Kovaleski DF, Zange SE, Antunes JLF. Estratificação sócio-econômica em estudos epidemiológicos de cárie dentária e doenças periodontais: características da produção na década de 90. Cad Saúde Pública. 2005;21(3):673-8.

7. Pitts N, Amaechi B, Niederman R, Acevedo A, Vianna R, Ganss C, et al. Global oral health inequalities: dental caries task group--research agenda. Adv Dent Res. 2011;23(2):211-20.

8. Schwendicke F, Dörfer CE, Schlattmann P, Foster Page L, Thomson WM, Paris S. Socioeconomic inequality and caries: a systematic review and meta-analysis. J Dent Res. 2015;94(1):10-8.

9. CDSS. Redução das desigualdades no período de uma geração: igualdade na saúde através da ação sobre os seus determinantes sociais: relatório final. Genebra: Comissão para os Determinantes Sociais da Saúde- OMS; 2010.

10. Moreira TP, Nations MK, Alves MSCF. Dentes da desigualdade: marcas bucais da experiência vivida na pobreza pela comunidade do Dendê, Fortaleza, Ceará, Brasil. Cad Saúde Pública. 2007;23(6):1383-92.

11. Peres K, De Magalhães Bastos ], Oliveira L. Severidade de cárie em crianças e relação com aspectos sociais e comportamentais. Rev Saúde Pública. 2000;34(4):402-8. 
12. Peres MA, Latorre MRDO, Sheiham A, Peres KG, Barros FC, Hernandez PG, et al. Determinantes sociais e biológicos da cárie dentária em crianças de 6 anos de idade: um estudo transversal aninhado numa coorte de nascidos vivos no Sul do Brasil. Rev Bras Epidemiol. 2003;6(4):293-306.

13. Baldani MH, Narvai PC, Antunes JLF. Cárie dentária e condições sócio-econômicas no Estado do Paraná, Brasil, 1996. Cad Saúde Pública. 2002;18(3): 755-63.

14. Gabardo M, Moyses S, Moysés S. Self-rating of oral health according to the oral health impact profile and associated factors: a systematic review. Rev Panam de Salud Pública. 2013;33(6):439-45

15. Silva SRC, Castellanos Fernandes RA. Autopercepção das condições de saúde bucal por idosos. Rev Saúde Pública. 2001;35(4):349-55.

16. Vale EB, Mendes ACG, Moreira RS. Autopercepção da saúde bucal entre adultos na região Nordeste do Brasil. Rev Saúde Pública. 2013;47(3):98-108.

17. Takagi D, Watanabe Y, Edahiro A, Ohara Y, Murakami M, Murakami K, et al. Factors affecting masticatory function of community-dwelling older people: Investigation of the differences in the relevant factors for subjective and objective assessment. Gerodont. 2017;34(3):357-64.

18. Slade GD, Nuttall N, Sanders AE, Steele JC, Allen PF, Lahti S. Impacts of oral disorders in the United Kingdom and Australia. Br Dent ]. 2005;198(8):489-93.

19. Barbato PR, Nagano HCM, Zanchet FN, Boing AF, Peres MA. Perdas dentárias e fatores sociais, demográficos e de serviços associados em adultos brasileiros: uma análise dos dados do Estudo Epidemiológico Nacional (Projeto SB Brasil 2002-2003). Cad Saúde Pública. 2007;23(8):1803-14.

20. Silva AER, Menezes AMB, Assunção MCF, Gonçalves H, Demarco FF, Vargas-Ferreira F, et al. Validation of selfreported information on dental caries in a birth cohort at 18 years of age. Plos One. 2014;9(9):1-8.

21. Chisini L, Cademartori M, Collares K, Tarquinio S, Goettems M, Demarco F, et al. Methods and logistics of an oral health cohort of university students from Pelotas, a Brazilian Southern city. Braz ] Oral Sc. 2019;18(e191460):1-15.

22. White IR, Royston P, Wood AM. Multiple imputation using chained equations: issues and guidance for practice. Statist Medic. 2011;30(4):377-99.

23. Aida ], Ando Y, Oosaka M, Niimi K, Morita M. Contributions of social context to inequality in dental caries: a multilevel analysis of Japanese 3-year-old children. Community Dent Oral Epidemiol. 2008;36(2):149-56.

24. Celeste RK, Nadanovsky P. How much of the income inequality effect can be explained by public policy? Evidence from oral health in Brazil. Health Policy. 2010;97(2-3):250-8.

25. Levin K, Davies C, Douglas G, Pitts N. Urban-rural differences in dental caries of 5-year old children in Scotland. Social Scienc Medic. 2010;71(11): 2020-7.

26. Monteiro CA, Mondini L, Costa RB. Mudanças na composição e adequação nutricional da dieta familiar nas áreas metropolitanas do Brasil (1988-1996). Rev Saúde Pública. 2000;34(3):251-8.

27. Piovesan C, Marquezan M, Kramer PF, Bönecker M, Ardenghi TM. Socioeconomic and clinical factors associated with caregivers' perceptions of children's oral health in Brazil. Community Dent Oral Epidemiol. 2011;39(3):260-7.

28. Silva SRCd, Rosell FL, Valsecki Júnior A. Percepção das condições de saúde bucal por gestantes atendidas em uma unidade de saúde no município de Araraquara, São Paulo, Brasil. Rev Bras Saúde Mater Infant. 2006;6(4):405-10.

29. Barreto SG. Associação da autopercepção de saúde bucal com parâmetros clínicos orais. Rev Bras odontol. 2011;68(2):268-73.

30. Araújo CS, Lima RC, Peres MA, Barros AJD. Utilização de serviços odontológicos e fatores associados: um estudo de base populacional no Sul do Brasil. Cad Saúde Pública. 2009;25(5):1063-72.

31. Vasconcelos LCA, Prado Júnior RR, Teles JBM, Mendes RF. Autopercepção da saúde bucal de idosos de um município de médio porte do Nordeste brasileiro. Cad Saúde Pública. 2012;28(6):1101-10.

32. Tosta TLD. A participação de estudantes universitários no trabalho produtivo e reprodutivo. Cad Pesqui. 2017;47(165):896-910.

33. Guerra MJC, Greco RM, Leite ICG, Ferreira EF, Paula MVQ. Impact of oral health conditions on the quality of life of workers. Ciênc Saúde Coletiva. 2014;19(12):4777-86.

34. Almeida TF, Couto MC, Oliveira MS, Ribeiro MB, Vianna MIP. Ocorrência de cárie dentária e fatores associados em crianças de 24 a 60 meses residentes em áreas cobertas pelo Programa Saúde da Família, em Salvador - BA, 2008. Rev Odontol UNESP. 2010;39(6):355-62.

35. Krieger $\mathrm{N}$. Theories for social epidemiology in the 21st century: an ecosocial perspective. Int ] Epidemiol. 2001;30(4):668-77.

36. Castilho ARF, Mialhe FL, Barbosa TS, Puppin-Rontani RM. Influência do ambiente familiar sobre a saúde bucal de crianças: uma revisão sistemática. J Pediatr. 2013;89(2):116-23.

37. Narvai P, Frazão P, Roncalli A, Antunes ]. Cárie dentária no Brasil: declínio, polarização, iniqüidade e exclusão social. Rev Panam Salud Publica. 2006;19(6):385-93. 